\title{
BMJ Open Association between parental socioeconomic status and offspring overweight/obesity from the China Family Panel Studies: a longitudinal survey
}

\author{
Suqin Ding $\left(\mathbb{0},{ }^{1,2}\right.$ Jingqi Chen, ${ }^{1}$ Bin Dong, ${ }^{1}$ Jie $\mathrm{Hu}^{3}$
}

To cite: Ding S, Chen J, Dong B, et al. Association between parental socioeconomic status and offspring overweight/ obesity from the China Family Panel Studies: a longitudinal survey. BMJ Open 2021;11:e045433. doi:10.1136/ bmjopen-2020-045433

- Prepublication history and additional materials for this paper are available online. To view these files, please visit the journal online (http://dx.doi. org/10.1136/bmjopen-2020045433).

Received 30 September 2020 Revised 26 February 2021 Accepted 12 March 2021

Check for updates

(C) Author(s) (or their employer(s)) 2021. Re-use permitted under CC BY-NC. No commercial re-use. See rights and permissions. Published by BMJ.

${ }^{1}$ Institute of Child and Adolescent Health, School of Public Health, Peking University, Beijing, China

${ }^{2}$ Department of Chronic Disease Control and Prevention, Suzhou Industrial Park Center for Disease Control and Prevention, Suzhou, China

${ }^{3}$ Menzies Health Institute Queensland, Griffith University, Brisbane, Queensland, Australia

\section{Correspondence to}

Dr Jingqi Chen;

g3jing_qi@bjmu.edu.cn and

Dr Bin Dong;

bindong@bjmu.edu.cn

\section{ABSTRACT}

Objective To examine the association between parental socioeconomic status (SES) and the risk of offspring overweight/obesity and the changes of the association that occur as children grow older.

Design We used data from the nationally representative Iongitudinal survey of the China Family Panel Studies of 2010 and its three follow-up waves in 2012, 2014 and 2016.

Participants A total of 6724 children aged $0-15$ years old were included.

Primary and secondary outcome measures Average household income and paternal and maternal education levels were used as SES indicators. Logistic regression model for panel data was used to examine the associations between SES indicators and child overweight/ obesity. A restricted cubic spline linear regression model was used to estimate body mass index (BMI) trajectories with child growth across parental SES levels.

Results Compared with the lowest education level (primary school or less), the ORs for fathers who had completed junior high school, senior high school and junior college or higher were 0.85 (95\% Cl 0.75 to 0.97$), 0.77$ (95\% Cl 0.64 to 0.92 ) and 0.72 (95\% Cl 0.55 to 0.93$)$, respectively. The corresponding 0 Rs for mothers were 0.76 ( $95 \% \mathrm{Cl} 0.67$ to 0.86$), 0.59$ (95\% Cl 0.47 to 0.72 ) and 0.45 (95\% $\mathrm{Cl} 0.34$ to 0.60$)$, respectively. A negative association between parental education and offspring overweight/obesity was observed in the first 10 years but not in children 11-15 years old. BMI differences across parental education levels emerged from birth and widened before 6-7 years old, but decreased before adolescence. High average household income was related to a low risk of offspring overweight/obesity but not when parental education level was adjusted for.

Conclusion High parental education levels were associated with a low risk of offspring overweight/obesity, especially before adolescence. Effective approaches need to be adopted in early childhood to reduce socioeconomic differences in overweight/obesity.

\section{INTRODUCTION}

There has been a substantial increase in childhood overweight/obesity in the past few decades. ${ }^{2}$ The global prevalence of obesity increased from $0.7 \%$ to $5.6 \%$ in girls and
Strengths and limitations of this study

- A nationally representative longitudinal dataset was used to estimate the association between parental socioeconomic status (SES) and offspring overweight/obesity and we also conducted subgroup analyses according to child's sex, residence, and baseline age groups.

- We used restricted cubic spline model to estimate body mass index trajectories with child growth across parental SES levels.

- Reported height and weight of children were used in this study.

- We did not adjust for dietary habits and physical activity factors among children who were under 10 years old.

from $0.9 \%$ to $7.8 \%$ in boys between 1975 and $2016 .{ }^{2}$ Numerous studies have reported that childhood overweight/obesity not only greatly increases risk of health problem in childhood,,$^{3-5}$ but also children with overweight/obese are at high risk of being overweight/obese in adulthood, which may contribute to chronic diseases and even premature mortality. ${ }^{6-9}$

Previous studies have made headway in identifying the association between parental socioeconomic status (SES) and offspring overweight/obesity. ${ }^{10-13}$ They have, however, arrived at different conclusions. For example, systematic reviews showed that in developed countries parents with higher SES were more likely to have a lower risk of offspring overweight/obesity ${ }^{10}$; conversely, in developing countries, higher parental SES was related to higher offspring overweight/obesity. ${ }^{11}$ However, a multicentre study conducted in Europe, which employed a standardised study protocol, showed heterogeneous associations between parental SES and offspring overweight in different countries. ${ }^{13}$ It seemed that the relationship varied depending on the 
economic development status of participants' residing country. ${ }^{10} 1113$

SES is often measured by indicators of income, education and occupation. Several studies have suggested that different indicators exert variable influences on childhood overweight/obesity through different pathways. ${ }^{14}$ For instance, education improves one's knowledge, values and problem-solving ability, occupation influences one's lifestyle and shared peer values, and income relates to resource access. ${ }^{14}$ Research has also shown that the association between parental SES and offspring overweight/ obesity varied depending on the SES indicators that were used. A review analysed 158 studies in high-income countries and found that $65.8 \%, 41.7 \%$ and $37.5 \%$ of the studies showed an inverse relationship using parental education, parental occupation and household income, respectively. ${ }^{10}$ It seemed that parental education was more consistently associated with offspring overweight/ obesity than the other indicators. ${ }^{14}$

Although a large number of studies have recognised parental socioeconomic differences in childhood overweight/obesity, what remains unclear is when the differences emerge and how they change across the offspring's age. A study in Belarus among children 0-7 years of age suggested that socioeconomic differences in body mass index (BMI) were present at birth and increased after 3 years. ${ }^{15}$ Howe et al found that socioeconomic differences in trajectories of obesity began at about 4 years in age and widened with the increasing age among children 2-10 years old in England. ${ }^{16}$ Bann et al ${ }^{17}$ reported that socioeconomic differences in BMI widened with age from 7 or 10 years to 15 years in the UK. These studies indicated a widening trend of socioeconomic differences in overweight/obesity as children grow older despite the different beginning ages. However, a systematic review found that negative relationships between parental SES and offspring obesity were less prevalent among children aged 12-18 years than those between 5 and 11 years. ${ }^{14}$ It seemed that socioeconomic differences may decrease in adolescence.

In China, studies indicated that the rate of overweight and obesity was positively associated with provinciallevel socioeconomic indicators. ${ }^{18}$ The socioeconomic differences in children's overweight/obesity seemed to increase between 1991 and 2006. ${ }^{19}$ Similar inconsistent results were found among studies that focused on families. A study among children aged 5-12 years in Guangzhou, China, suggested that both household per capita income and maternal education levels were positively associated with childhood overweight/obesity. ${ }^{12}$ Conversely, another study among children aged 0-6years old found that parents' lower education levels were associated with a higher risk of child obesity ${ }^{20}$; a study failed to identify a significant association between family SES and BMI in children aged 7-11 years old. ${ }^{21}$ However, most of these research projects were cross-sectional studies in local cities. Therefore, this study with a longitudinal nationally representative dataset may provide important insights on the associations between parental SES and offspring overweight/obesity in China.

In order to determine what socioeconomic determinants take the main responsibility for the differences in childhood overweight/obesity and find out the onset and development of the differences, we examined the associations between offspring overweight/obesity and parental SES, and the changes of BMI over children's ages across parental SES.

\section{METHODS}

\section{Participants}

The data used in this study were sourced from the China Family Panel Studies (CFPS), ${ }^{22}$ which was carried out by the Institute of Social Science Survey of Peking University. ${ }^{23}{ }^{24}$ The CFPS was designed to be a nationally representative longitudinal survey and focused on factors such as subjects' economic situation, education level, health status and so on at the individual, family and community levels. The baseline was conducted in 2010 using a multistage probability sampling strategy. Twenty-five provinces were included, representing $94.5 \%$ of the total population of China. Since 2010, there have hitherto been four follow-up waves in the CFPS (2012, 2014, 2016 and 2018) and the databases from 2010 to 2016 are publicly available. New households and individuals were incorporated into each wave because of the change of family structure and formation of new families. Informed consent was obtained from all participants. Details have been described in previous work. ${ }^{23}{ }^{24}$ All members in selected households were interviewed. Those who were aged 0-15 years old, were interviewed at least twice $(\mathrm{n}=10017)$, and had complete data for height and weight, household income, paternal and maternal education, child sex, child age, and residence were included in this study. As a result, a total of 6724 children were included for analysis.

\section{Outcome measures}

The height and weight of the children were reported by either their parents or guardians. Studies have examined the validity of self-reported height and weight in assessing the obesity status of children, which suggested an almost perfect agreement with the measured values. ${ }^{25}{ }^{26} \mathrm{An}$ article published in Lancet reported that self-reported BMI was highly correlated with measured BMI (correlation coefficient 0.96 ) from the evidence of 538 country-years with both types of measurements available. ${ }^{1}$ Therefore, self-reported height and weight can be used in large-scale epidemiological studies when the measurement is not practical. Participants' BMI is their weight in kilograms divided by the square of their height in metres. Since BMI cut-off points for overweight and obesity by the International Obesity Task Force do not apply to children under 2 years of age, we used age-specific and sex-specific WHO growth reference for children aged 0-5 years ${ }^{27}$ and 5-19 years. ${ }^{28}$ According to the BMI-for-age Z-score (BAZ) cutoff points by $\mathrm{WHO}, \mathrm{BAZ}>+2 \mathrm{SD}$ and $\mathrm{BAZ}>+3 \mathrm{SD}$ indicate 
overweight and obesity, respectively, for children under 5 years old, and BAZ $>+2$ SD indicates obesity for children aged 5-19 years old..$^{29}$ However, in this study children were tracked from under 5 years to over 5 years, and to avoid confusion by adopting two different cut-off points, we used +2SD as the cut-off point to define overweight/ obese $(\mathrm{BAZ}>+2 \mathrm{SD})$ and non-overweight/obese (BAZ $\leq+2 \mathrm{SD})$.

\section{Socioeconomic status}

SES is often measured by indicators of income, education and occupation. Since occupation is an unordered categorical variable, some comprehensive indexes were constructed to rank occupations; however, some of them were derived from international average education and income ${ }^{30}$ which might not be suitable for the situation in China, and some of them were subjective indicators. Therefore, the average household income and paternal and maternal education levels were taken as the SES indicators in this study.

The CFPS conducted a very detailed survey of household income, including working wages, assets, monetary gifts, pensions and any other forms of subsidy. Average household income was calculated as the total household income divided by the number of family members. Considering the large economic disparities present in different regions in China, we converted the average household income into a relative variable using the reported average household income divided by provincial-level household per capita annual disposable income of urban and rural in each survey year, ${ }^{31}$ which was further classified into the following five quintiles: poorest, lower $20 \%$, medium $20 \%$, higher $20 \%$ and richest. The paternal and maternal education levels were divided into four categories, namely primary school or less, junior high school, senior high school and junior college or higher.

\section{Covariates}

Covariates in this study included child's age, sex, birth weight, dietary habits and physical exercise, family residence (urban vs rural) and paternal/maternal obesity $\left(\mathrm{BMI} \geq 28 \mathrm{~kg} / \mathrm{m}^{2}\right)$. The children's age at baseline was divided into three groups of $0-1,2-10$ and $11-15$ years old when examining the association between parental SES and childhood overweight/obesity by different age groups. Birth weight of the child was recalled by parents or guardians and categorised as low (birth weight $<2500 \mathrm{~g}$ ), normal (2500 g $\leq$ birth weight $<4000 \mathrm{~g}$ ) or high (birth weight $\geq 4000 \mathrm{~g}$ ). Dietary habits and physical exercise were self-reported by the children; however, only children over 10 years old were asked these questions. Dietary habits were estimated from three questions: 'Did you have meat last week?', 'Did you have fresh fruits and vegetables last week?', 'Did you have fried food last week'. Physical exercise was estimated by the question 'In the latest month which is not a vacation, how often did you participate in physical exercise' in 2010 and 2012, and the options include 'almost every day', '2-3 times a week',
'2-3 times a month', 'once a month' and 'never'. Another question, 'How many times did you participate in physical exercise last week', was used in 2014 and 2016. In order to be consistent, we classified children as participating in physical exercise 'more than 2-3 times a week' and 'less than twice a week'.

\section{Statistical analysis}

Stata V.13.0 was used to analyse the data. A descriptive exploratory analysis was carried out to identify the characteristics of participants. Logistic regression of randomeffects model was performed for panel data to estimate the OR and $95 \%$ CI for the association between parental SES and offspring overweight/obesity. Demographic factors, including child sex, age and residence, ${ }^{32} 33$ were adjusted for, while average household income and paternal/maternal education were further adjusted for. Subgroup analyses were also conducted by child's sex, residence and baseline age.

We estimated the BMI trajectories across different parental SES groups from 0 to 15 years old using a restricted cubic spline regression model to allow nonlinear patterns. Four knot points setting at 1, 6, 10 and 14 years for boys and 1, 6, 10 and 15 years for girls were identified by the spline models. The knot points were considered to be reasonable according to a previous study using similar models. ${ }^{16}$ Linear spline regression of randomeffects models for the panel data was used to examine whether BMI differences across different parental SES levels changed with child growth. Models of average household income, paternal education and maternal education were constructed separately. The new variables derived by spline models were included and were also used to assess the interaction with average household income or paternal/maternal education. ${ }^{34}$ Given that the interaction terms in the average household income model were not statistically significant in either boys or girls, they were not included in the final model. The 95th percentile $\left(\mathrm{P}_{95}\right)$ of the predicted BMI by age is shown by line graphs.

We performed a sensitivity analysis by further adjusting for potential confounders that were associated with childhood overweight/obesity, including child's sex, age, residence, birth weight, average household income, paternal and maternal obesity, and child's dietary habits and physical exercise (online supplemental file 1). ${ }^{335-37}$ A p value $<0.05$ with two sides was considered statistically significant.

\section{Patient and public involvement}

This research was done without patient and public involvement.

\section{RESULTS}

A total of 6724 children were followed up for 2-6years, with a median follow-up duration of 4.0 (IQR: 2-6) years. The numbers of children who were interviewed two, three and four times were 3047, 2276 and 1401, respectively. 
Table 1 Baseline characteristics of children and parents

\begin{tabular}{ll}
\hline Variable & Value (N=6724) \\
\hline Child sex, n (\%) & \\
$\quad$ Boys & $3500(52.1)$ \\
Girls & $3224(48.0)$ \\
\hline Residence, n (\%) & \\
$\quad$ Urban & $2739(40.8)$ \\
$\quad$ Rural & $3985(59.2)$ \\
$\begin{array}{l}\text { Baseline age (years), mean } \\
\text { (SD ) }\end{array}$ & $5.7(4.0)$ \\
\hline
\end{tabular}

Paternal education level, n (\%)

\begin{tabular}{|c|c|}
\hline Primary school or less & 2272 (33.8) \\
\hline Junior high school & $2819(41.9)$ \\
\hline Senior high school & 1000 (14.9) \\
\hline Junior college or higher & $633(9.4)$ \\
\hline \multicolumn{2}{|c|}{ Maternal education level, n (\%) } \\
\hline Primary school or less & $2978(44.3)$ \\
\hline Junior high school & 2440 (36.3) \\
\hline Senior high school & $764(11.4)$ \\
\hline Junior college or higher & $542(8.1)$ \\
\hline \multicolumn{2}{|c|}{ Average household income ( $¥)$, median (IQR), mean } \\
\hline 2010 & $4662(2583,8433), 7280$ \\
\hline 2012 & 6667 (3075, 11 400), 8995 \\
\hline 2014 & 7900 (3986, 13 333), 10520 \\
\hline 2016 & 8220 (3990, 14 690), 11971 \\
\hline Birth weight (kg), mean (SD) & $3.2(0.54)$ \\
\hline
\end{tabular}

$I Q R$, interquartile range.

The median number of surveys was 3 (IQR: 2-3). The sample size came to 4371 in 2010, 5049 in 2012, 4857 in 2014, and 4249 in 2016. The baseline characteristics of the children and parents are presented in table 1 .

Children in families that are in the medium $20 \%$ quintile of average household income seemed less likely to be overweight/obese than those in the poorest families, with an OR of 0.85 (95\% CI 0.74 to 0.99 ) (table 2). After adjusting for covariates, the medium $20 \%$ quintile, as well as those in the richest quintile, still had a negative association with overweight/obesity, independent of children's age, sex, residence and survey year; the ORs were 0.79 (95\% CI 0.68 to 0.92 ) and 0.80 (95\% CI 0.69 to 0.93). However, after further adjustment for paternal and maternal education levels, the associations among all the income groups were not significant.

Paternal and maternal education levels showed a consistent gradient of negative associations with their children's overweight/obesity (table 2). The effect estimates were even stronger after adjustment for covariates. This result indicates that higher parental education levels were associated with a lower risk of offspring overweight/ obesity (all $\mathrm{p}_{\text {trend }}<0.001$ ), independent of child's age, sex and residence. Compared with the lowest education level, after adjusting for all covariates, the ORs for fathers who had completed junior high school, senior high school and junior college or higher were 0.85 (95\% CI 0.75 to 0.97 ), 0.77 (95\% CI 0.64 to 0.92$)$ and 0.72 (95\% CI 0.55 to 0.93$)$, respectively. The corresponding ORs for mothers were 0.76 (95\% CI 0.67 to 0.86 ), 0.59 (95\% CI 0.47 to 0.72 ) and 0.45 (95\% CI 0.34 to 0.60$)$, respectively. The results showed that paternal education was reversely associated with childhood overweight/obesity, largely independent of maternal education, average household income and other demographic factors of child, and the same results were found for maternal education. Compared with the same levels of education on the paternal side, improvements in maternal education levels seemed to have a stronger effect on offspring overweight/obesity.

We also examined the parental SES effect on offspring overweight/obesity in the subgroups. Figure 1 indicates that the association patterns between average household income and childhood overweight/obesity were different among the subgroups. After adjusting for the child's age, compared with those in the poorest families, urban boys in the medium 20\% and higher $20 \%$ quintiles and urban girls in the higher $20 \%$ quintile were less likely to be obese, whereas no significant difference was found in rural boys and girls. Figure 2 shows a significant gradient of negative associations between paternal and maternal education levels and offspring overweight/obesity in urban girls and rural boys and girls after adjusting for age. The risk of overweight/obesity significantly decreased with higher parental education levels $\left(\mathrm{p}_{\text {trend }}<0.001\right.$ ). In urban boys, however, only parents who had completed junior college or a higher education level showed significance.

The associations between parental SES and offspring overweight/obesity by children's baseline age groups are shown in table 3. After adjusting for child sex, residence and survey year, inverse associations between average household income and childhood overweight/obesity were found in the medium $20 \%$ quintile in the $0-1$ years group and in the richest quintile in the 2-10 years group. A significant gradient of negative associations was observed in different paternal and maternal education levels among the $0-1$ years and $2-10$ years groups (all $\left.\mathrm{p}_{\text {trend }}<0.001\right)$ but not in the $11-15$ years group.

Spline models were used to estimate BMI trajectories across parental SES over child's age (figures 3 and 4 and online supplemental tables $\mathrm{S} 1-\mathrm{S} 3$ ). The $\mathrm{P}_{95}$ of predicted BMI of the richest and medium 20\% quintiles was lower than the poorest quintile in boys (figure 3); however, there was no significant difference in girls. Figure 4 shows that the $\mathrm{P}_{95}$ of BMI disparities across paternal and maternal education levels varied from childhood to adolescence in both boys and girls. BMI disparities across parental education widened from birth until about $6-7$ years in age and then narrowed until 11 years in boys and $12-13$ years in girls. The disparities thereafter crossed and inversed in adolescence. Children whose parents with higher education levels had lower $\mathrm{P}_{95}$ of predicted BMIs from 
Table 2 Association between parental SES and offspring overweight/obesity

\begin{tabular}{llll}
\hline & Unadjusted OR & Adjusted OR & Adjusted OR† \\
\hline Quintile of average household income & & & Ref \\
Poorest & Ref & Ref & $0.89(0.77-1.03)$ \\
Lower 20\% & $0.88(0.76-1.02)$ & $0.86(0.74-1.00)$ & $0.87(0.75-1.02)$ \\
Medium 20\% & $0.85(0.74-0.99)$ & $0.79(0.68-0.92)$ & $1.02(0.87-1.18)$ \\
Higher 20\% & $1.04(0.90-1.21)$ & $0.89(0.76-1.03)$ & $0.95(0.81-1.11)$ \\
Richest & $1.01(0.87-1.16)$ & $0.80(0.69-0.93)$ & 0.923
\end{tabular}

Paternal education level

\begin{tabular}{llll} 
Primary school or less & Ref & Ref & Ref \\
Junior high school & $0.93(0.83-1.05)$ & $0.77(0.68-0.87)$ & $0.85(0.75-0.97)$ \\
Senior high school & $0.74(0.62-0.87)$ & $0.61(0.51-0.72)$ & $0.77(0.64-0.92)$ \\
\hline Junior college or higher & $0.58(0.47-0.72)$ & $0.46(0.37-0.57)$ & $0.72(0.55-0.93)$ \\
$P_{\text {trend }}$ & $<0.001$ & $<0.001$ & 0.001
\end{tabular}

Maternal education level

\begin{tabular}{llll} 
Primary school or less & Ref & Ref & Ref \\
\hline Junior high school & $0.95(0.84-1.06)$ & $0.71(0.62-0.80)$ & $0.76(0.67-0.86)$ \\
Senior high school & $0.68(0.57-0.82)$ & $0.51(0.42-0.62)$ & $0.59(0.47-0.72)$ \\
\hline Junior college or higher & $0.55(0.44-0.69)$ & $0.37(0.30-0.47)$ & $0.45(0.34-0.60)$ \\
$P_{\text {trend }}$ & $<0.001$ & $<0.001$ & $<0.001$ \\
\hline
\end{tabular}

$P_{\text {trend }}$ value represents test of linear trends across five quintiles of average household income or parental education levels.

${ }^{*}$ Adjusted for child sex, age and residence. Survey year was also added for average household income.

†Adjusted for child sex, age, residence, survey year, average household income, paternal education level and maternal education level except itself.

Ref, reference; SES, socioeconomic status.

childhood to preadolescence; however, the results were then inverse in adolescence. Additionally, the disparities were greater among girls than among boys in preadolescence but were smaller in adolescence.

Findings of sensitivity analysis showed that the associations between paternal/maternal education level and offspring's overweight/obesity were similar after adjusting for child's sex, residence, age, birth weight, average household income, and paternal and maternal obesity (online supplemental tables S4 and S5).

\section{DISCUSSION}

To our knowledge, the present study provides novel insight into the associations between parental SES and offspring overweight/obesity using nationally representative longitudinal data in China. These data confirmed that high levels of parental education are associated with a low risk of offspring overweight/obesity, especially before the children are 10 years of age. However, the association was not significant in children who are $11-15$ years old. BMI differences in children across parental education levels emerged from birth and widened before they reached 6-7 years of age and then decreased before adolescence.

The gradient of negative association between parental education and offspring overweight/obesity before adolescence has been well established in our study. The impact of parental education on offspring overweight/ obesity was stronger in girls than in boys; this result was consistent with a previous study in Japan. ${ }^{38}$ We also further substantiated that parental education has a much stronger effect on offspring overweight/obesity and is more representative as an SES indicator in its relationship with overweight/obesity than income is. The way in which parental education could exert an influence on offspring overweight/obesity is complex. First, education raises health literacy, which enables parents to access available information to make decisions and take actions that positively affect their children's health. ${ }^{39}$ Second, studies have shown that high parental education levels are positively associated with their children's healthy lifestyles. ${ }^{40} 41$ Third, education is strongly linked to employment opportunity, and therefore income, which may affect their ability to afford healthier food and other health-related resources. $^{42}$ Therefore, improving parental education levels is likely to reduce risk of childhood overweight/ obesity. Additionally, parents with lower education levels should be given priority in public health measures implementation, such as health education.

BMI disparities across parental education levels widened from birth until about 6-7 years in age, which is in 


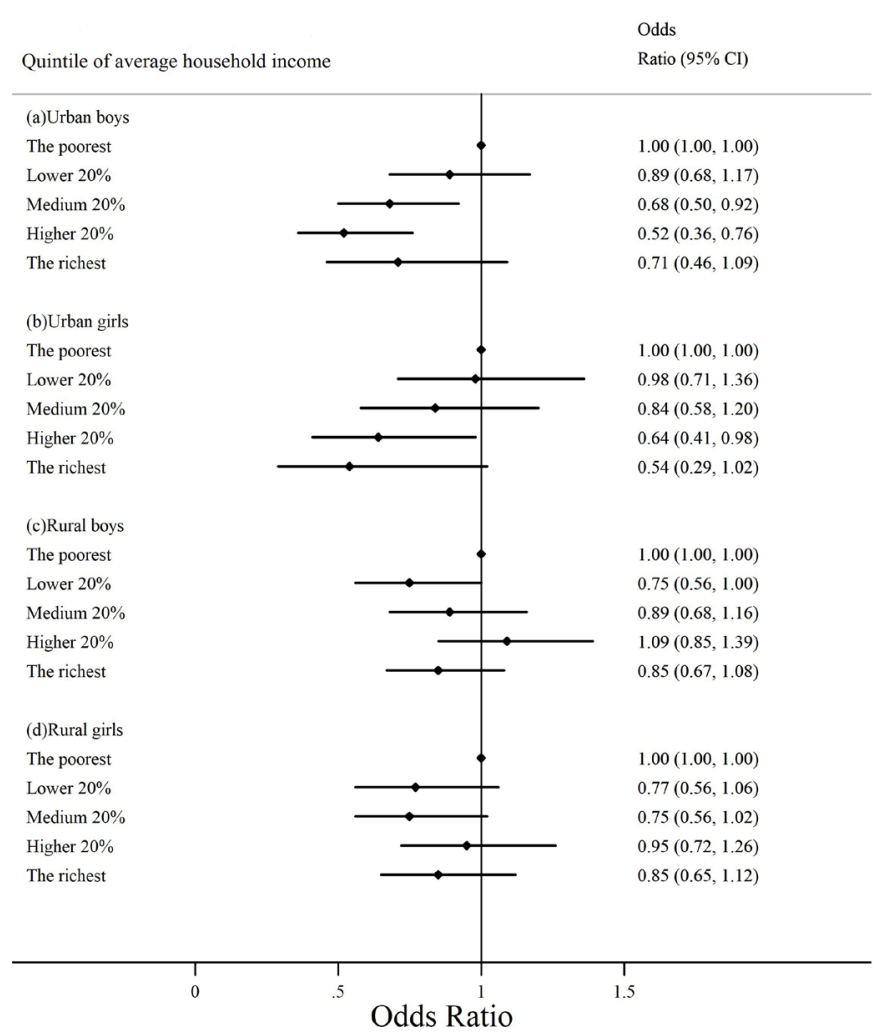

Figure 1 Association between average household income and offspring overweight/obesity by subgroups, with the poorest as reference, adjusted for child age and survey year.

agreement with previous studies among children younger than 10 years. ${ }^{1516}$ However, the disparities narrowed once children grew to be older than 10 years. This was inconsistent with other studies which showed the continuous widening trend of socioeconomic differences in BMI. ${ }^{17} \mathrm{~A}$ possible explanation for this might be due to differences in the statistical approach used, as the interaction terms we added in the models allowed for different interaction shapes in their respective age periods. Additionally, ethnic differentials might be another possible explanation. A study suggested that the relationship between parental education and BMI was weaker in East Asia than in North American and Australia. ${ }^{43}$

Significant negative associations were observed in different parental education levels in children younger than 10 years, but no association was found in those aged 11-15 years. This result appears to support the narrowing trend of BMI differences. A systematic review also reported that fewer negative relationships between parental SES and offspring overweight/obesity were found among children aged $12-18$ years than those aged 5-11 years. ${ }^{14}$ $\mathrm{Fu}$ and George, ${ }^{44}$ using longitudinal data from the China Health and Nutrition Survey (CHNS), found that parental education had little or marginal effect on the age trajectories of overweight in children who are 6-18 years old. Various parenting practices may lead to overweight/obesity disparities in early childhood; however, as children grow, adolescents are more likely to spend more time with their peers and be influenced by their peers in terms of dietary habits and physical activities. ${ }^{45}$ Findings of this study highlight the importance of public health initiatives in early childhood to reduce socioeconomic differences in childhood overweight/obesity.

The association between family income and offspring overweight/obesity was not consistent. In urban areas, children in medium-income families seemed less likely to be overweight/obese and those in the richest families had the same risk as those in the poorest families, while the associations were not significant in rural children. High family income does not necessarily mean the family members have more health knowledge or lead a healthier lifestyle. A systematic review showed no association between income and children's healthy or unhealthy dietary patterns in medium-income countries. ${ }^{46}$ China has been undergoing rapid socioeconomic changes, and the economic prosperity, urbanisation and supermarket
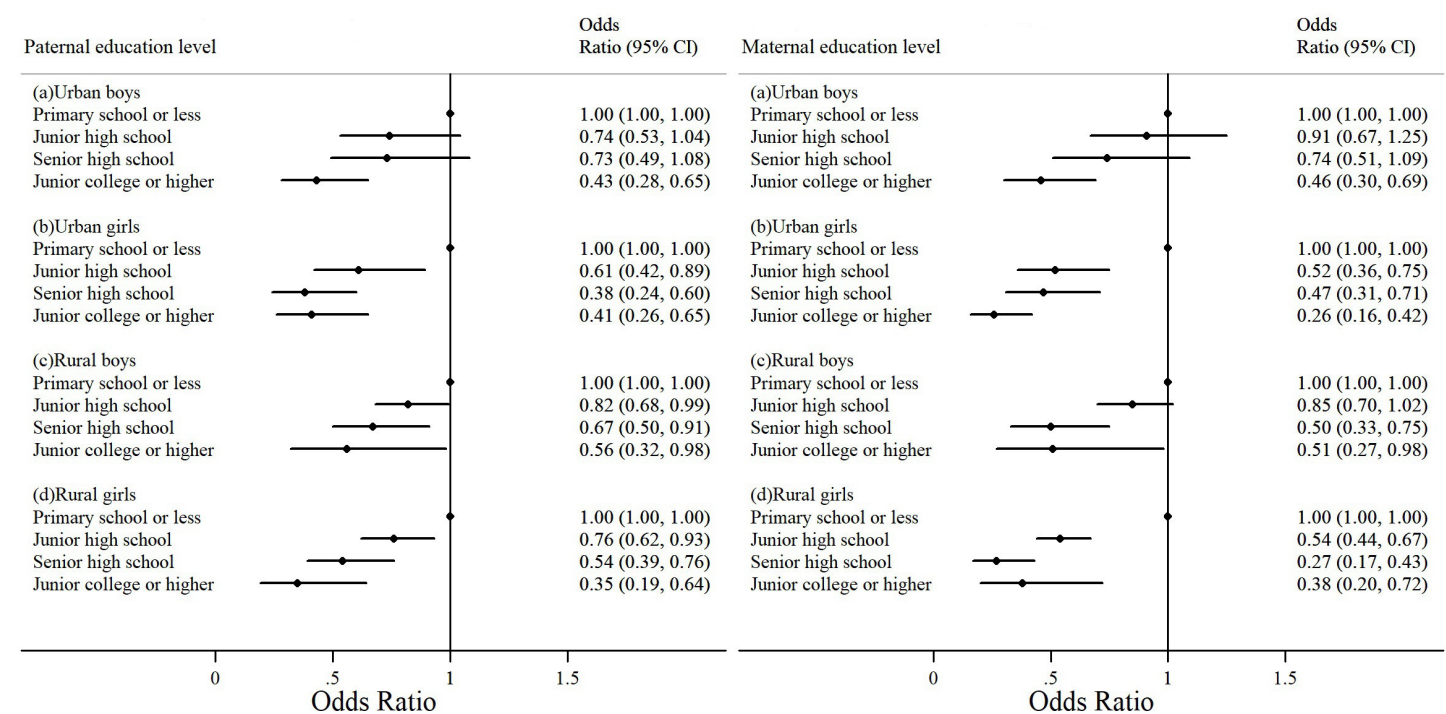

Figure 2 Association between parental education level and offspring overweight/obesity by subgroups, with primary school or less education level as reference, adjusted for child age. 
Table 3 Association between parental SES and offspring overweight/obesity by child baseline age groups

\section{Unadjusted OR Adjusted OR*}

$\begin{array}{lllll}0-1 & 2-10 & 11-15 & 0-1 & 2-10\end{array}$

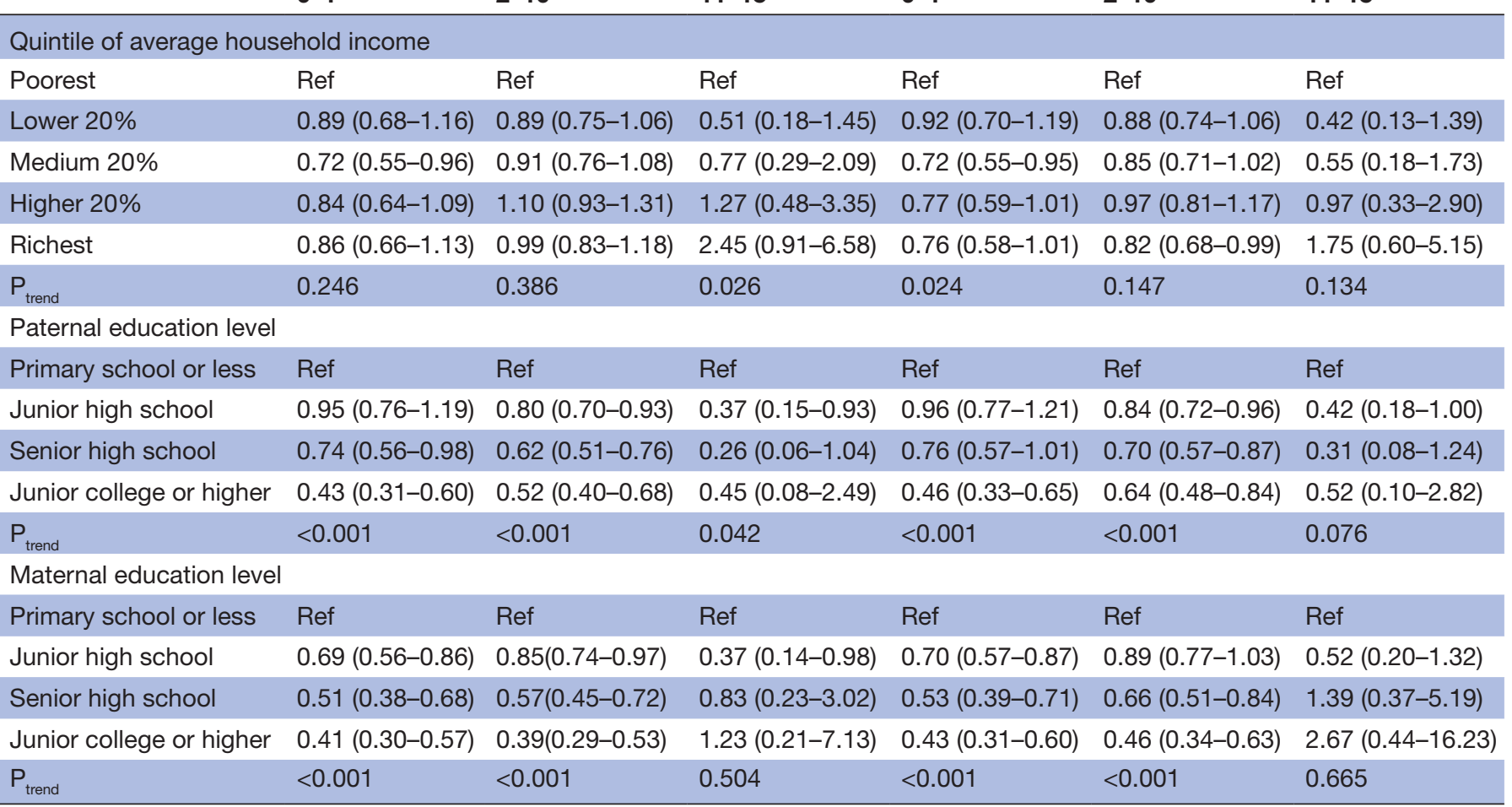

$\mathrm{P}_{\text {trend }}$ value represents test of linear trends across five quintiles of average household income or parental education levels.

*Adjusted for child sex and residence. Survey year was also added for average household income.

Ref, reference.

expansion lead to rapid shifts towards unhealthy dietary pattern (such as fat-rich diet, fast food, sugar-sweetened beverages) and reduced physical activity. ${ }^{478}$ Income and food price are two main factors that influence the families' food choice. High income may increase access to supermarkets and allow for higher consumption of energydense foods. ${ }^{46}$ Along with socioeconomic development, the unhealthy diet trends may shift towards the poor and

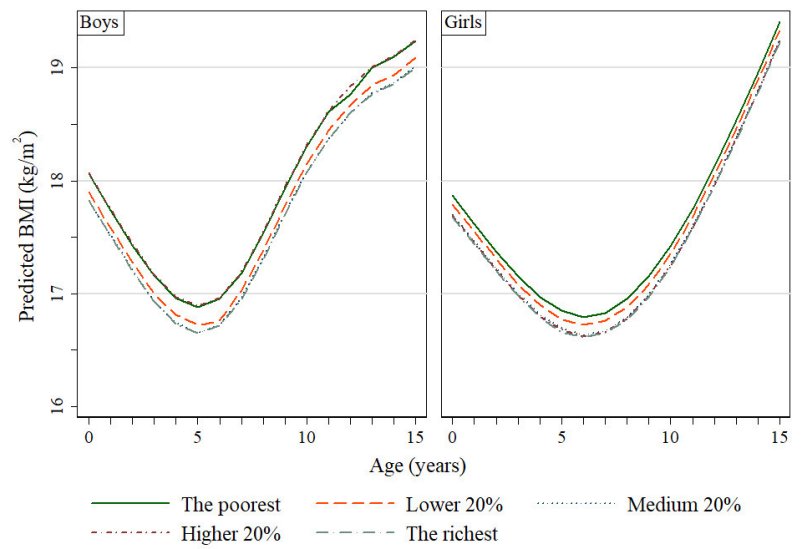

Figure $3 \mathrm{P}_{95}$ of predicted $\mathrm{BMI}$ from 0 to 15 years by category of average household income. Adjusted for residence and survey year. BMI, body mass index; $P_{95}$, 95th percentile. rural people, and those who with low SES tend to adopt unhealthier diets and to be overweight/obese. ${ }^{46} 48$

The findings need to be interpreted in light of several limitations. First, parent-reported (or guardian-reported) height and weight of children were used in this study. In order to examine the validity of the reported data, we compared the data with the measured data from other nationally representative surveys. In this study, the prevalence of obesity among children aged $7-15$ years

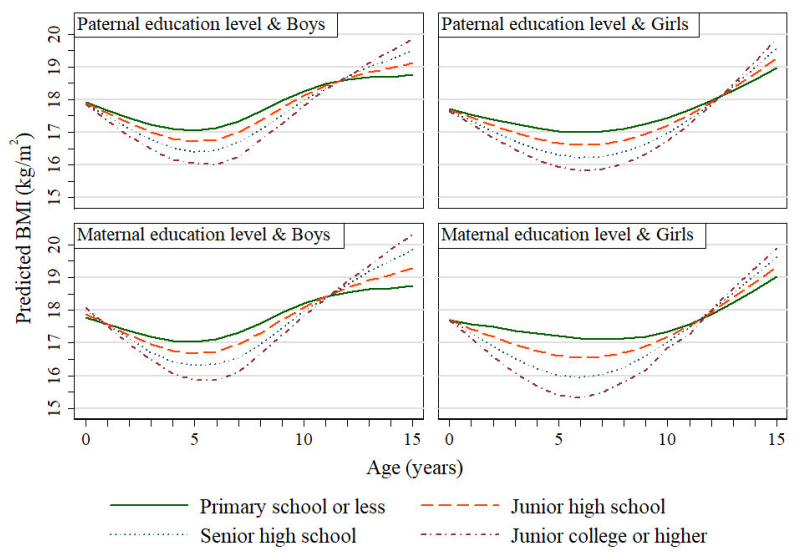

Figure $4 \mathrm{P}_{95}$ of predicted BMI from 0 to 15 years by category of parental education level. Adjusted for residence and survey year. BMI, body mass index; $\mathrm{P}_{95}$, 95th percentile. 
was $10.9 \%$. Data from the Chinese National Survey on Students' Constitution and Health showed the prevalence of obesity among children aged $7-15$ years ranged from $1.2 \%$ to $10.5 \%$ across different age and sex groups in 2010. ${ }^{32}$ The CHNS indicated that the prevalence of obesity among children aged $6-17$ was $9.32 \%$ in 2011 and $10.75 \%$ in $2015 .^{49}$ The BMI trajectories of children aged 0-15 years old were almost the same as the measured results in Pan et al's study. ${ }^{50}$ It seems that the biases of the reported data were acceptable. Second, we did not adjust for the dietary habits and physical activity factors among children who were under 10 years old because the information was not collected in the survey. In addition, although we adjusted for several sociodemographic characteristics in the analysis, the possibility of residual confounding factors could not be fully eliminated. Despite these limitations, due to a large sample from a nationally representative longitudinal survey, a substantial association between parental SES and offspring overweight/obesity was identified.

\section{CONCLUSION}

Associations are identified between parental SES and offspring overweight/obesity from the CFPS. The findings indicate the importance of parental education on offspring overweight/obesity, especially in children aged $0-10$ years. This study may help identify the families with high risk of offspring overweight/obesity, and effective approaches need to be adopted in early childhood to reduce overweight/obesity for those families. From a long-term point of view, promoting universal education level in the society to improve parents' health literacy, parenting skills and healthy lifestyle may play important role in addressing childhood overweight/obesity.

Acknowledgements We are grateful to the 985 Program of Peking University and the National Natural Science Foundation of China for funding and the Institute of Social Science Survey of Peking University for carrying out the initial studies and sharing the data.

Contributors SD: data curation and analysis, writing the original draft and editing. JC: supervision and reviewing. BD: data analysis, reviewing and editing. JH: reviewing and editing. All authors contributed to the final draft and finally approved it for publication.

Funding The China Family Panel Studies was funded by the 985 Program of Peking University and the National Natural Science Foundation of China (71450001).

Competing interests None declared.

Patient consent for publication Not required.

Ethics approval All CFPS surveys were approved by Peking University Institutional Review Board.

Provenance and peer review Not commissioned; externally peer reviewed.

Data availability statement Data are available in a public, open access repository. The data that support the findings of this study are publicly released and can be accessed through Peking University Open Research Data at http://dx.doi.org/10. 18170/DVN/45LCSO.

Supplemental material This content has been supplied by the author(s). It has not been vetted by BMJ Publishing Group Limited (BMJ) and may not have been peer-reviewed. Any opinions or recommendations discussed are solely those of the author(s) and are not endorsed by BMJ. BMJ disclaims all liability and responsibility arising from any reliance placed on the content. Where the content includes any translated material, BMJ does not warrant the accuracy and reliability of the translations (including but not limited to local regulations, clinical guidelines, terminology, drug names and drug dosages), and is not responsible for any error and/or omissions arising from translation and adaptation or otherwise.

Open access This is an open access article distributed in accordance with the Creative Commons Attribution Non Commercial (CC BY-NC 4.0) license, which permits others to distribute, remix, adapt, build upon this work non-commercially, and license their derivative works on different terms, provided the original work is properly cited, appropriate credit is given, any changes made indicated, and the use is non-commercial. See: http://creativecommons.org/licenses/by-nc/4.0/.

ORCID iD

Suqin Ding http://orcid.org/0000-0001-9087-0963

\section{REFERENCES}

$1 \mathrm{Ng} \mathrm{M}$, Fleming T, Robinson M, et al. Global, regional, and national prevalence of overweight and obesity in children and adults during 1980-2013: a systematic analysis for the global burden of disease study 2013. Lancet 2014;384:766-81.

2 Abarca-Gómez L, Abdeen ZA, Hamid ZA. Worldwide trends in body-mass index, underweight, overweight, and obesity from 1975 to 2016: a pooled analysis of 2416 population-based measurement studies in 128.9 million children, adolescents, and adults. Lancet 2017;390:2627-42

3 Egan KB, Ettinger AS, Bracken MB. Childhood body mass index and subsequent physician-diagnosed asthma: a systematic review and meta-analysis of prospective cohort studies. BMC Pediatr 2013;13:121.

4 Griffiths LJ, Parsons TJ, Hill AJ. Self-esteem and quality of life in obese children and adolescents: a systematic review. Int J Pediatr Obes 2010:5:282-304.

5 Meo SA, Altuwaym AA, Alfallaj RM, et al. Effect of obesity on cognitive function among school adolescents: a cross-sectional study. Obes Facts 2019;12:150-6.

6 Sommer A, Twig G. The impact of childhood and adolescent obesity on cardiovascular risk in adulthood: a systematic review. Curr Diab Rep 2018;18:91.

7 Geng T, Smith CE, Li C, et al. Childhood BMI and adult type 2 diabetes, coronary artery diseases, chronic kidney disease, and cardiometabolic traits: a Mendelian randomization analysis. Diabetes Care 2018;41:1089-96.

8 Llewellyn A, Simmonds M, Owen CG, et al. Childhood obesity as a predictor of morbidity in adulthood: a systematic review and metaanalysis. Obes Rev 2016;17:56-67.

9 Reilly JJ, Kelly J. Long-term impact of overweight and obesity in childhood and adolescence on morbidity and premature mortality in adulthood: systematic review. Int J Obes 2011;35:891-8.

10 Barriuso L, Miqueleiz E, Albaladejo R, et al. Socioeconomic position and childhood-adolescent weight status in rich countries: a systematic review, 1990-2013. BMC Pediatr 2015;15:129.

11 Dinsa GD, Goryakin Y, Fumagalli E, et al. Obesity and socioeconomic status in developing countries: a systematic review. Obes Rev 2012;13:1067-79.

12 Liu W, Liu W, Lin R, et al. Socioeconomic determinants of childhood obesity among primary school children in Guangzhou, China. BMC Public Health 2016;16:482.

13 Bammann K, Gwozdz W, Lanfer A, et al. Socioeconomic factors and childhood overweight in Europe: results from the multi-centre IDEFICS study. Pediatr Obes 2013;8:1-12.

14 Shrewsbury V, Wardle J. Socioeconomic status and adiposity in childhood: a systematic review of cross-sectional studies 1990-2005. Obesity 2008;16:275-84.

15 Patel R, Tilling K, Lawlor DA, et al. Socioeconomic differences in childhood BMI trajectories in Belarus. Int J Obes 2018;42:1651-60.

16 Howe LD, Tilling K, Galobardes B, et al. Socioeconomic disparities in trajectories of adiposity across childhood. Int $J$ Pediatr Obes 2011;6:e144-53.

17 Bann D, Johnson W, Li L, et al. Socioeconomic inequalities in childhood and adolescent body-mass index, weight, and height from 1953 to 2015: an analysis of four longitudinal, observational, British birth cohort studies. Lancet Public Health 2018;3:e194-203.

18 Dong Y, Jan C, Ma Y, et al. Economic development and the nutritional status of Chinese school-aged children and adolescents from 1995 to 2014: an analysis of five successive national surveys. Lancet Diabetes Endocrinol 2019;7:288-99. 
19 He W, James SA, Merli MG, et al. An increasing socioeconomic gap in childhood overweight and obesity in China. Am J Public Health 2014;104:e14-22.

20 Yang HH, Chen XX, Meng J. Survey on the prevalence of obesity in 0 to 6 -year-old Beijing urban children. Chinese $J$ Child Health Care 2009;17:694-7.

21 Wei DM, LJ W, Gao AY, et al. Study on the relations among the screen-based sedentary behaviors, family factors and body mass index of children. J Peking University 2015;47:390-4.

22 Institute of Social Science Survey, Peking University. China family panel studies (CFPS). Peking University Open Research Data Platform 2015

23 Xie Y, Lu P. The sampling design of the China family panel studies (CFPS). Chin J Sociol 2015;1:471-84.

$24 \mathrm{Xie}$ Y, Hu J. An introduction to the China family panel studies (CFPS) Chinese Sociol Rev 2014;47:3-29.

$25 \mathrm{Kee} \mathrm{CC,} \mathrm{Lim} \mathrm{KH,} \mathrm{Sumarni} \mathrm{MG,} \mathrm{et} \mathrm{al.} \mathrm{Validity} \mathrm{of} \mathrm{self-reported} \mathrm{weight}$ and height: a cross-sectional study among Malaysian adolescents. BMC Med Res Methodol 2017;17:85.

26 Pérez A, Gabriel K, Nehme EK, et al. Measuring the bias, precision, accuracy, and validity of self-reported height and weight in assessing overweight and obesity status among adolescents using a surveillance system. Int J Behav Nutr Phys Act 2015;12:S2.

27 World Health Organization. Child growth standards, 2007. Available: https://www.who.int/childgrowth/software/en/ [Accessed Aug 2019]

28 World Health Organization. Growth reference 5-19 years, 2007. Available: https://www.who.int/growthref/who2007_bmi_for_age/en/ [Accessed Aug 2019].

29 de Onis M, Lobstein T. Defining obesity risk status in the general childhood population: which cut-offs should we use? Int J Pediatr Obes 2010;5:458-60.

30 Duncan OD. Occupations and social status. New York: The Free Press, 1961.

31 National Bureau of Statistics. China statistical yearbook 2011-2017. Available: http://www.stats.gov.cn/tjsj/ndsj/ [Accessed Aug 2019].

32 Song $\mathrm{Y}$, Wang $\mathrm{H}-\mathrm{J}$, Dong $\mathrm{B}$, et al. 25-year trends in gender disparity for obesity and overweight by using WHO and IOTF definitions among Chinese school-aged children: a multiple cross-sectional study. BMJ Open 2016;6:e011904.

33 Song Y, Ma J, Wang H-J, et al. Secular trends of obesity prevalence in Chinese children from 1985 to 2010: urban-rural disparity. Obesity 2015;23:448-53.

34 Luo JF, Jin H, BY L, et al. The application of restricted cubic Spline in nonlinear regression. Chinese J Health Stat 2010;27:229-32.

35 Woo Baidal JA, Locks LM, Cheng ER, et al. Risk factors for childhood obesity in the first 1,000 days: a systematic review. Am J Prev Med 2016;50:761-79.
36 Heslehurst N, Vieira R, Akhter Z, et al. The association between maternal body mass index and child obesity: a systematic review and meta-analysis. PLoS Med 2019;16:e1002817.

37 Wang Y, Cai L, Wu Y, et al. What childhood obesity prevention programmes work? A systematic review and meta-analysis. Obes Rev 2015;16:547-65.

38 Mizuta A, Fujiwara T, Ojima T. Association between economic status and body mass index among adolescents: a community-based cross-sectional study in Japan. BMC Obes 2016;3:47.

39 Morrison AK, Glick A, Yin HS. Health literacy: implications for child health. Pediatr Rev 2019;40:263-77.

40 Fernández-Alvira JM, te Velde SJ, De Bourdeaudhuij I, et al. Parental education associations with children's body composition: mediation effects of energy balance-related behaviors within the ENERGYproject. Int J Behav Nutr Phys Act 2013;10:80.

41 Mostafavi F, Moradi G, Azadi N, et al. Using Oaxaca decomposition to study socioeconomic inequity of physical activity among children aged 10-12 years: a study in West of Iran. Int J Prev Med 2019;10:83.

42 Quick V, Eck KM, Delaney C, et al. Stability of differences in weightrelated characteristics of mothers across economic, cultural, social, and Environmental-Health indicators of socioeconomic status. Int J Environ Res Public Health 2019;16 doi:10.3390/ ijerph16203866

43 Silventoinen K, Jelenkovic A, Latvala A, et al. Parental education and genetics of BMI from infancy to old age: a pooled analysis of 29 twin cohorts. Obesity 2019;27:855-65.

44 Fu Q, George LK. Sex, socioeconomic and regional disparities in age trajectories of childhood BMI, underweight and overweight in China. Asian Popul Stud 2015;11:134-48.

45 World Health Organization. WHO skills for health. skills-based health education including life skills: an important component of a childfriendly/health-promoting school.

46 Hinnig PdeF, Monteiro JS, de Assis MAA, et al. Dietary patterns of children and adolescents from high, medium and low human development countries and associated socioeconomic factors: a systematic review. Nutrients 2018;10 doi:10.3390/nu10040436

47 Popkin BM, Adair LS, Ng SW. Global nutrition transition and the pandemic of obesity in developing countries. Nutr Rev 2012;70:3-21.

48 Popkin BM. Global nutrition dynamics: the world is shifting rapidly toward a diet linked with noncommunicable diseases. Am J Clin Nutr 2006;84:289-98.

49 Ma SJ, Zhang YQ, Yang L, et al. [Analysis on the trend of overweight and obesity of children and adolescents in 9 provinces of China from 1991 to 2015]. Zhonghua Yu Fang Yi Xue Za Zhi 2020;54:133-8.

50 Pan $\mathrm{H}$, Jiang $\mathrm{Y}$, Jing $\mathrm{X}$, et al. Child body mass index in four cities of East China compared to Western references. Ann Hum Biol 2009;36:98-109. 